http://jmscr.igmpublication.org/home/

ISSN (e)-2347-176x ISSN (p) 2455-0450

crossref DOI: https://dx.doi.org/10.18535/jmscr/v10i2.27

\author{
Dournal Of Medical Science And Clinical Research \\ IGM Publication \\ An Official Publication of IGM Publication
}

\title{
Role of Preoperative Embolisation in Endoscopic Excision of Juvenile Nasopharyngeal Angiofibroma
}

Authors

\section{K. Athiyaman M.S. ENT ${ }^{1^{*}}$, M. Gowri Shankar M.S. ENT D.L.O, DNB ${ }^{2}$, Vineeth. $\mathbf{S}^{3}$}

${ }^{1}$ Associate Professor of Otorhinolaryngology and Head and Neck Surgery, Department of Otorhinolaryngology and Head and Neck Surgery, Govt Stanley Medical College and Hospital, Chennai-600001

${ }^{2}$ Professor and Head of department, Department of Otorhinolaryngology and Head and Neck Surgery, Govt Stanley

Medical College and Hospital, Chennai-600001

${ }^{3}$ Post graduate in M.S. ENT, Department of Otorhinolaryngology and Head and Neck Surgery, Govt Stanley Medical College and Hospital, Chennai-600001

*Corresponding Author

K. Athiyaman M.S. ENT

Associate Professor of Otorhinolaryngology and Head and Neck Surgery, Department of Otorhinolaryngology and Head and Neck Surgery, Govt Stanley Medical College and hospital, Chennai-600001

Abstract
Juvenile nasopharyngeal angiofibroma is a unique benign vascular tumor accounting for less than $1 \%$ all head
and neck tumors which is almost exclusively present in adolescent males. Objective of this study is to bring before
the role of preoperative embolisation in endoscopic excision of juvenile nasopharyngeal angiofibroma at a tertiary
care hospital. This is a retrospective study from the clinical data from the patients treated for JNA tumors from
$2017-2020$ at a tertiary care hospital. The tumour was staged according to Fisch system and clinical presentation,
local examination, nasal endoscopy findings, radiological findings, approach of surgical resection, complications
and recurrence were noted. In our study, we have found that among total 10 cases, mean age of presentation was
15.8 years and about $80 \%$ of the patients had stage I or stage II disease. Preoperative embolisation was carried
out in all patients 24 hours prior to surgery. The endoscopic endonasal approach was choice of surgery in all.
Intraoperative blood loss was found to be around 380 ml. In conclusion, Preoperative embolisation has reduced
the blood loss during surgery and need for multiple blood transfusions and has reduced ICU stay post operatively.
Keywords: Juvenile Nasopharyngeal Angiofibroma; Preop embolization; Endoscopic endonasal surgery;
Rhinology.

\section{Introduction}

Juvenile nasopharyngeal angiofibroma (JNA) is a unique rare benign vascular lesion of skull, accounting for less than $1 \%$ all head and neck tumors. JNA ${ }^{(1)}$ is an aggressive proliferative disease of mesenchymal origin which arises in the cranial base in the spheno-pterygoid junction which involves the root of the human masticatory and respiratory apparatus. Even though this tumour is classified as benign lesion histologically, the bone eroding property of JNA is it's hallmark which allows its extension to pterygoid, the clivus and the sphenoid sinus floor $^{(2)}$

JNA predominately present in the male population, most commonly seen between 10 and 25 years of age. The diagnosis of JNA is purely based on the clinical and radiology findings as the preoperative tissue diagnosis is not advisable. The most common symptoms are nasal obstruction and epistaxis ${ }^{(3)}$. Nasal obstruction is unilateral initially which later becomes bilateral due to the 
nasopharyngeal extension and septal deviation by the expanding lesion. Epistaxis is unprovoked, intermittent and profuse. Less common symptoms are nasal discharge, facial pain, facial swelling, proptosis, sinusitis, anosmia and conductive hearing loss due to the eustachian tube involvement. Ophthalmoplegia is rare due to extension into cavernous sinus and orbital apex ${ }^{(4)}$. On endoscopic examination, JNA appears as rubbery hyper vascular mass with smooth surface present behind the tail of the middle turbinate filling the choana or completely obstructing the nasopharynx. Tumor spreads distinctly from the site of origin which helps in its differentiation from other lesions of skull base such as schwannoma, hemangioma, meningioma, hemangiopericytoma and sarcoma. JNA most commonly originates from the superior margin of sphenopalatine foramen and spreads along the natural foramina and fissures. The lesion may infiltrate the submucosa or erode the surrounding bony structures. It spreads to involve the pterygomaxillary fossa, infratemporal fossa, pterygoid plates, orbit, cavernous sinus, foramen lacerum and carotid canal.

Fisch staging system for the spread of JNA has 4 stages as described below.

\begin{tabular}{|l|l|}
\hline Stage 1 & $\begin{array}{l}\text { Tumour limited to the nasopharyngeal cavity; bone destruction negligible or limited to } \\
\text { the sphenopalatine foramen }\end{array}$ \\
\hline Stage 2 & $\begin{array}{l}\text { Tumour invading the pterygopalatine fossa or the maxillary, ethmoid or sphenoid } \\
\text { sinus with bone destruction }\end{array}$ \\
\hline Stage 3a & $\begin{array}{l}\text { Tumour invading the infratemporal fossa or orbital region: without intracranial } \\
\text { involvement }\end{array}$ \\
\hline Stage 3b & $\begin{array}{l}\text { Tumour invading the infratemporal fossa or orbital region: with intracranial extradural } \\
\text { (parasellar) involvement }\end{array}$ \\
\hline Stage 4a & $\begin{array}{l}\text { Intracranial intraduraltumour: without infiltration of the cavernous sinus, pituitary } \\
\text { fossa or optic chiasma }\end{array}$ \\
\hline Stage 4b & $\begin{array}{l}\text { Intracranial intraduraltumour: with infiltration of the cavernous sinus, pituitary fossa } \\
\text { or optic chiasma }\end{array}$ \\
\hline
\end{tabular}

The management of juvenile angiofibroma is challenging because of the complex anatomy of the skull base and the young age of the affected population. Combined with the higher rates of residual or recurrent lesions in minimal surgical approaches and facial growth disturbances, cosmetic and functional complications in extended external approaches the management of juvenile angiofibroma is a challenge even to this day. The advent of endoscopic approaches and preoperative embolization along with latest techniques like radiofrequency ablation has greatly evolved the management of juvenile angiofibroma. In this study, we present an overview of the main features of juvenile angiofibroma and current strategies in its management.

\section{Materials and Methods}

The present, retrospective study included 10 patients who underwent surgery for JNA tumors between January 2017 to November 2020 in our hospital. The protocol of the investigation was approved by the institutional Review Board and informed consent was obtained from patients prior to their inclusion in the study. Inclusion criteria for our study were all Cases of JNA admitted to our institute during the period between January 2017 to November 2020. The exclusion criteria were patients with severe systemic disease or with any bleeding disorders.

The following data were evaluated for all patients like age of presentation, preoperative symptoms, duration of symptoms, staging of disease, intraoperative blood loss, ICU stay and ventilatory support and postoperative complications. Patients were evaluated by a detailed clinical examination followed by endoscopic examination and Enhanced CT and MRI scans were done to confirm the location and size of the tumor as a preoperative diagnosis. Preoperative imaging was also used for tumor 
staging and was staged according to Fisch system. Additionally, Digital Subtraction Angiography was performed to know the feeding vessel of tumor.All patients enrolled in the study underwent surgical treatment and underwent preoperative embolisation 24 hours before surgery using poly vinyl alcohol (PVA) particles. Endoscopic endonasal approach was the choice of surgical approach in all. Resected specimens were sent for histopathological analysis and diagnosis confirmed. Intraoperative blood loss and postoperative complications were analyzed and the cases are on continuous follow up.

\section{Results}

In our study there were total of 10 patients, all of whom were males. The mean age at the point of surgery was 15.8 years (range 10-20years) (Table I : Age distribution). All the patients were found to be between the ages of 10 and 20 years in our study.

The most common symptoms of JNA in our study were nasal obstruction (80\%), epistaxis (60\%), nasal discharge (50\%), facial swelling (30\%), headache $(30 \%)$, change in voice $(50 \%)$, ear pain/reduced hearing(40\%) and anosmia (20\%). The most common symptom of JNA was nasal obstruction (80\%). Though no orbital involvement was noted in our study it has been noted in other studies.

Table I: Presenting symptoms of patients in our study

\begin{tabular}{|l|c|}
\hline Symptoms & $\begin{array}{c}\text { Number of patients } \\
\text { (percentage) }\end{array}$ \\
\hline Nasal obstruction & $8(80 \%)$ \\
\hline Epistaxis & $6(60 \%)$ \\
\hline Nasal discharge & $5(50 \%)$ \\
\hline Facial swelling & $3(30 \%)$ \\
\hline Headache & $3(30 \%)$ \\
\hline Voice change & $5(50 \%)$ \\
\hline Ear pain/reduced hearing & $4(40 \%)$ \\
\hline Anosmia & $2(20 \%)$ \\
\hline
\end{tabular}

Patients presented to the hospital with varying duration of symptoms. In our study, duration of illness before presenting to hospital was $<6$ months in 2 patients(20\%), 6 months to 1 year in 5 patients (50\%) and between 1-2 years in 3 patients
(30\%) of patients. Most frequent was between 6 months to 1 year duration. Diagnostic nasal endoscopy was done for all cases, with which nasal cavity and nasopharynx were examined thoroughly for evidence of any mass.

Preoperatively all our patients were assessed with CT and MRI imaging to evaluate the location of each tumor (Figure .2). The tumors were staged according to Fisch staging system. Fisch staging system describes the characteristics of tumour growth and invasion, especially the analysis of extension at the skull base, which makes this system a practical guide to the surgical approach and we found that $50 \%$ cases were stage $1,30 \%$ were stage 2 and $20 \%$ cases belonged to stage 3, there were no stage 4 disease in our study.
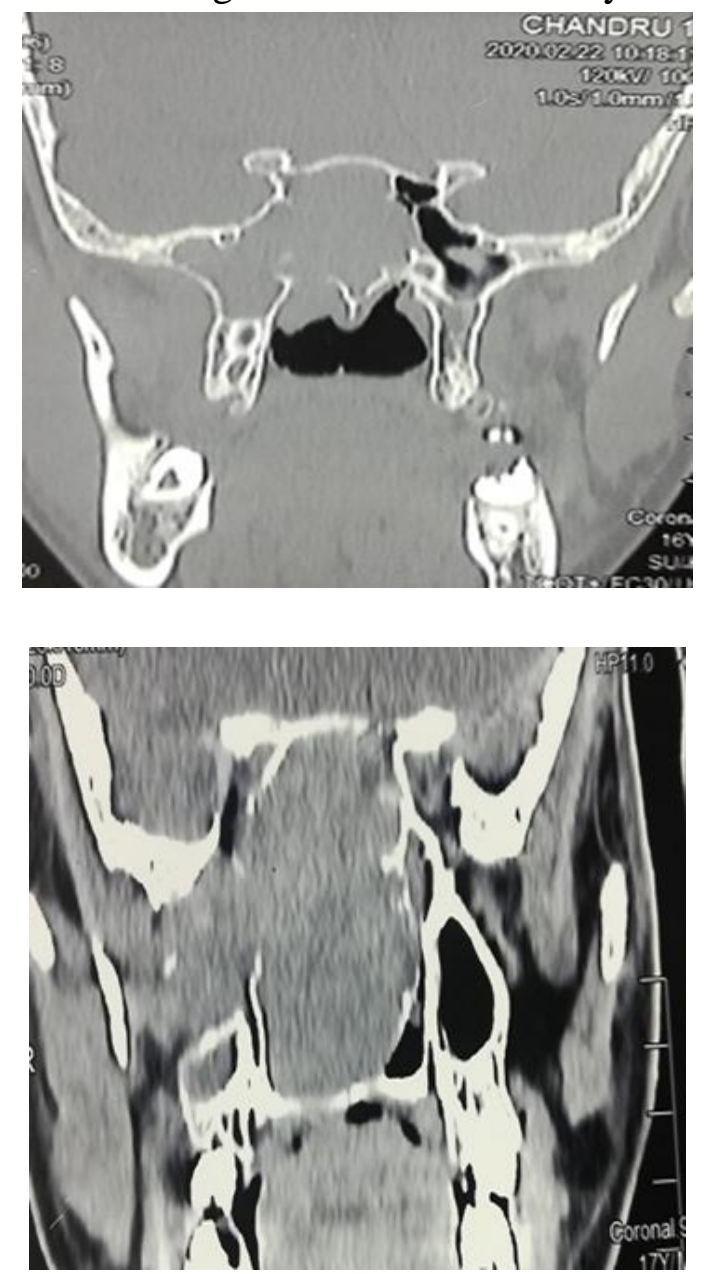

Figure 1: Coronal computed tomography (CT) scan of nose and paranasal sinuses: JNA occupying nasopharynx extending into the right nasal fossa 
In all cases presented to our hospital, preoperative embolization was done 24 hours before surgery. Endonasal endoscopic surgery was the choice of surgery in all patients. Surgical time taken varied according to stage of disease from 90mins for stage I to 4 hours for stage III. Intraoperative blood loss was analyzed in each case. The intraoperative blood loss was calculated by the blood collected in suction machine which was measured using a measuring cup and also by counting gauze pieces used during surgery which was weighed pre and post operatively. It was found to be an average blood loss of $220 \mathrm{ml}$ for stage I, $383 \mathrm{ml}$ for stage II and $775 \mathrm{ml}$ for stage III (Table II- intraoperative blood loss). An average blood loss of $380 \mathrm{ml}$ was found which when compared to intraop blood loss from other studies was similar to results found in Roger $\mathrm{G}^{(5)}(350 \mathrm{ml})$ which had $45 \%$ of cases at stage 3 (Radkowski) and Cloutier $^{(6)}(380 \mathrm{ml})$ which had $70 \%$ of cases at stage 3(Radkowski) while being significantly better than Ardehali ${ }^{(7)}$ (770 ml) which had $8 \%$ of cases at stage 3(Radkowski) and Bhalla and Narayanaswamy $^{(8)}(612 \mathrm{ml})$ with $17 \%$ cases at stage 3or higher(Fisch)

Table II: Intraoperative blood loss and surgical time taken in JNA tumor surgery in our study

\begin{tabular}{|l|c|c|c|}
\hline $\begin{array}{l}\text { Case } \\
\text { number }\end{array}$ & $\begin{array}{c}\text { Stage of disease } \\
\text { (fisch staging) }\end{array}$ & $\begin{array}{c}\text { Surgical time taken } \\
\text { (In hours })\end{array}$ & $\begin{array}{c}\text { Intra op blood loss } \\
\text { (in ml) }\end{array}$ \\
\hline 1 & I & 1.5 & $200 \mathrm{ml}$ \\
\hline 2 & II & 2.5 & $400 \mathrm{ml}$ \\
\hline 3 & I & 2.0 & $250 \mathrm{ml}$ \\
\hline 4 & I & 1.5 & $200 \mathrm{ml}$ \\
\hline 5 & III & 4.0 & $750 \mathrm{ml}$ \\
\hline 6 & II & 2.5 & $350 \mathrm{ml}$ \\
\hline 7 & I & 1.5 & $200 \mathrm{ml}$ \\
\hline 8 & I & 2.0 & $250 \mathrm{ml}$ \\
\hline 9 & III & 4.0 & $800 \mathrm{ml}$ \\
\hline 10 & II & 3.0 & $400 \mathrm{ml}$ \\
\hline
\end{tabular}

All patients were extubated in OT and did not require any post op intubation and kept in observation in ICU for 1 day post operatively and shifted toward the next day. No patient required blood transfusion. The tumor specimens were sent for histopathological examination in all cases. In all cases histopathological evaluation confirmed the features of JNA.

There were no major postoperative complications like post operative hemorrhage, CSF leak, septicemia, breathing difficulty or complications of embolisation in any of the patients. There was no need for any ventilator support post operatively. Post operatively there were two cases which developed minor complications like synechiae and which was treated post op. Crusting was present in almost all cases but septum was preserved and no septal perforation was found in follow up. All these patients were followed up for a year, with regular endoscopic examination. There was one incidence of recurrence which was diagnosed after one year. Tumour was found to be in stage 1 and was treated with endonasal endoscopic surgery.

\section{Discussion}

Angiofibroma was first described by Hippocrates in the $5^{\text {th }}$ century, but term was coined by Friedberg in $1940^{(9)}$. According to the previous studies in the literature, tumor affects the male population in the adolescent age group. Similarly, in our study, mean age of presentation was 15 years and affecting males. The high prevalence of tumor in the male population is explained by various theories, immunohistological and genetic studies $^{(10)}$.

Juvenile angiofibroma most commonly arises from the superior margin of sphenopalatine foramen. It has frequently been termed 'nasopharyngeal angiofibroma' because of its 
early submucosal expansion towards the nasopharynx, Due to this location, nasal obstruction and nasal bleeding may be the presenting symptoms. JNA is a vascular malformation from the remnants of first branchial arch. This explains site of origin and blood supply, tissue architecture, cellular \& molecular key stones ${ }^{(11)}$.

According to Nicolai et $\mathrm{al}^{(12)}$, usual presentation of patients will be progressive nasal obstruction (80\%), recurrent epistaxis (40\%) and swelling of the cheek. Similarly, in this present study, nasal obstruction and epistaxis were the common symptoms.

Diagnosis of JNA is usually based on the clinical presentation and radiological imaging studies. Contrast enhanced computed tomography (CT) is ideal to recognize the origin, extensions as well as pattern of vascularity of the lesion and helps to differentiate it from sinonasal polyp, neurofibroma and nasopharyngeal carcinoma. In 1981, Sessions first introduced the Classification for Staging of tumor. Many authors like Fisch, Chandler and Andrews adapted their several staging systems ${ }^{(13)}$. Our study classified tumours based on fisch classification. Fisch staging system describes the characteristics of tumour growth and invasion, especially the analysis of extension at the skull base, which makes this system a practical guide to the surgical approach

JNA tumors occur in the anterior skull base and this location results in difficulties in the diagnosis and surgical treatment. Surgical resection is the modality of treatment in management of JNA. Various approaches like transpalatine, lateral rhinotomy, transpalatine combined with transnasal and endoscopic surgery have been described. Endonasal endoscopic approach is safe and easier approach in Stage I or Stage II and also feasible in extensive lesions in the hands of experienced surgeon.Preoperative embolisation of juvenile nasopharyngeal angiofibromas (JNA) has been shown to reduce operative times and blood loss. Although traditional transarterial (TA) embolization is commonly used, direct tumoral puncture (DTP) has also been advocated as an alternative recently ${ }^{(14)}$.

All cases in our study were treated by Transnasal endoscopic approach with preoperative embolisation without adjuvant radiotherapy. After admission patient underwent pre operative embolisation in Digital Substraction Angiography unit of department of radiodiagnosis to see the arterial feeders and angioarchitecture of the tumour. Embolisation was then done by selective cathetarisation by interventional radiologist. Embolization was done using PVA particles (Polyvinyl alcohol foam)of size (300-500 micrometer). Availability of preoperative embolisation caused reduction in the vascularity of tumor significantly and improved the intraoperative handling of tumor by reducing intraoperative bleeding. Though embolisation is a procedure that can have a multitude of complications ranging from common complications like sore throat, hemifacial pain and paresthesia, nausea and vomiting, headache, neck pain, TMJ pain, fever, periorbital pain, hematoma in the femoral region, lower limb pain, reversible change in visual acuity, dyspnea, dysphagia, tongue paraesthesia and major complications like common iliac artery thrombosis, acute pulmonary oedema, facial paralysis, stroke, necrosis of the tip of the tongue and facial atrophy, no embolisation complications were reported in our study. Within $24 \mathrm{hrs}$ patients then underwent surgery to have maximum effect of embolization.

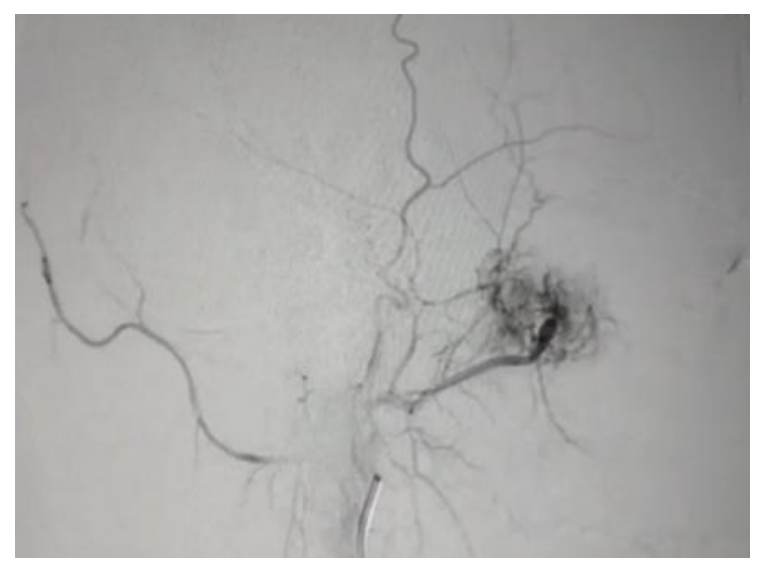




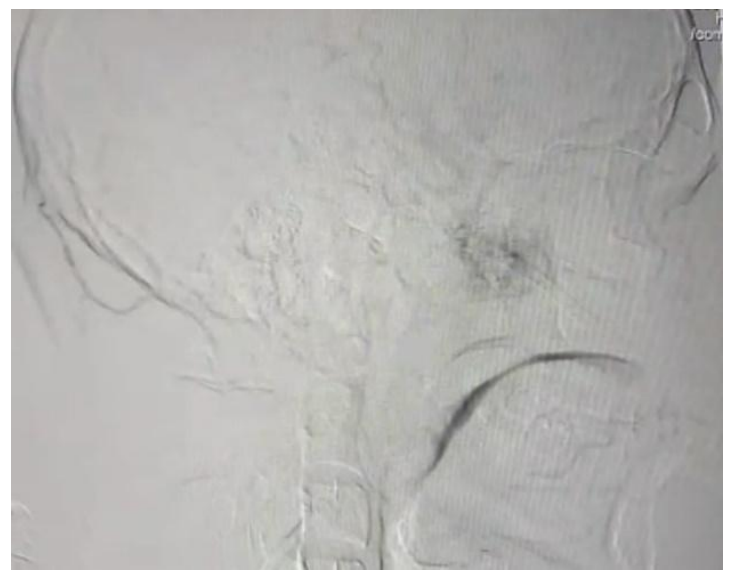

Figure 2: Angiogram showing pre and post embolisation of tumour using PVA foam

Endonasal Endoscopic surgery with preoperative embolisation is fast becoming the choice of approach by ENT surgeons worldwide. It is because of the magnified view of surgical field it provides, better control of bleeding and complete clearance of tumor thereby reducing the recurrence rate ${ }^{(15,16,17)}$. Other benefits include reduced surgical time and hospitalization, no external scars and complications like trismus, watering of eyes and facial deformity can be avoided $^{(18)}$.

In this present study, Intraoperative and postoperative period was uneventful without any complications. All patients were on continuous follow up for nasal endoscopic examination at monthly interval and a repeat scan in selective cases was performed at six monthly intervals. In a study by Pradip Kumar et al ${ }^{(19)}$ and Sharfuddin Mahmud et al, recurrence rate was $12.5 \%$ and $10 \%$ respectively. Similarly, in present study, we had one patient with recurrence after a year. Recurrent case was managed with surgery without any adjuvant radiotherapy.

\section{Conclusion}

Endonasal Endoscopic approach with preoperative embolisation is a suitable and safe approach for vascular tumors like JNA. Preoperative embolisation in patients undergoing resection of juvenile angiofibroma is not devoid of complications but rare. Preop embolization in juvenile nasopharyngeal angiofibroma has significantly reduced the blood loss during surgery and need for multiple blood transfusions and has reduced the duration of post operative ICU stay.

\section{Acknowledgements}

Financial Support- None

Conflict of Interest- None

Ethical Standards- Institutional Ethical Committee Approval Obtained

Informed Consent- Taken From All Patients

\section{References}

1. Tan L. Loh T. Benign and Malignant Tumors of the Nasopharynx: Cummings Otolaryngology: Head and Neck Surgery. 6th edition. Canada: Elsevier Saunders, 2015.p1420- 1421.

2. Schick B, Kahle G. Radiological findings in angiofibroma. Acta Radiologica. 2000; 41(6): 585-593

3. Schick B. Juvenile Angiofibroma. ScottBrown's Otorhinolaryngology Head\& Neck Surgery. 8th ed. Boca Raton, FL: CRC Press; 2018.

4. Safadi A, Schreiber A, Fliss D, Nicolai P. Juvenile Angiofibroma: Current Management Strategies. Journal of Neurological Surgery Part B: Skull Base. 2018;79(01):021-030.

5. Roger G, Tran Ba Huy P, Froehlich P, Van Den Abbeele T, Klossek JM, Serrano E, et al. Exclusively endoscopic removal of juvenile nasopharyngeal angiofibroma: trends and limits. Arch Otolaryngol Head Neck Surg. 2002; 128(8):928-35

6. Cloutier T, Pons Y, Blancal JP, Sauvaget E, Kania R, Bresson D et al. Juvenile nasopharyngeal angiofibroma: does the external approach still make sense? Otolaryngol Head and Neck Surg.2012; 147

7. Ardehali MM, Samimi Ardestani SH, Yazdani N, Goodarzi H, Bastaninejad S. Endoscopic approach for excision of juvenile nasopharyngeal angiofibroma: 
complications and outcomes. Am J Otolaryngol. 2010; 31(5):343-9 (5): 95863.

8. Bhalla 2018 Juvenile NA, Juvenile Nasopharyngeal Angiofibroma: Changing Paradigms in Management, Vijay Bhalla and Ramakrishnan Narayanaswamy, Bengal Journal of Otolaryngology and Head Neck Surgery, 2018, 26, pages\{35$42\}$

9. Ibrahim W, Abduljawad H. Juvenile Nasopharyngeal Angiofibroma: Imaging Characteristics and Pre-Operative Embolization. Bahrain Medical Bulletin. 2016;38(4):230-232.

10. Mishra S, Praveena NM, Panigrahi RG, Gupta YM. Imaging in the diagnosis of juvenile nasopharyngeal angiofibroma Journal of Clinical Imaging Science. 2013;3:1.

11. Schick B, Urbschat S. New aspects of pathogenesis of juvenile angiofibroma. Hospital Medicine 2004; 65(5): 269-273.

12. Nicolai P, Schreiber A, Bolzoni Villaret A. Juvenile Angiofibroma: Evolution of Management. International Journal of Pediatrics. 2012;2012:1-11.

13. Somayaji N, Talwade R, Shukla A, Pramila V, Nagesh R. Multidetector Computed Tomography Evaluation of Juvenile Nasopharyngeal Angiofibroma. The Journal of Medical Sciences. 2016;2(2):38-41.

14. Mishra S, Praveena NM, Panigrahi RG, Gupta YM. Imaging in the diagnosis of juvenile nasopharyngeal angiofibroma Journal of Clinical Imaging Science. 2013;3:1.

15. Andrade N, Pinto J, de Oliveira Nóbrega M, Aguiar J, Aguiar T, Vinhaes É. Exclusively Endoscopic Surgery for Juvenile Nasopharyngeal Angiofibroma. Otolaryngology-Head and Neck Surgery. 2007;137(3):492-496.
16. Mohammadi Ardehali M, Samimi Ardestani S, Yazdani N, Goodarzi H, Bastaninejad S. Endoscopic approach for excision of juvenile nasopharyngeal angiofibroma: complications and outcomes. American Journal of Otolaryngology. 2010;31(5):343-349.

17. Nicolai P, Berlucchi M, Tomenzoli D et al (2003) Endoscopic surgery for juvenile angiofibroma: when and how. Laryngoscope 113(5):775-782.

18. Mahmud S, Biswas D, Aich M, Sardar M. Management of Juvenile Nasopharyngeal Angiofibroma: A Three Years Retrospective Study. Bangladesh Journal of Otorhinolaryngology. 2015;21(1), pp.33-37.

19. Tiwari P, Teron P, Saikia N, Saikia H, Bhuyan U, Das D. Juvenile Nasopharyngeal Angiofibroma: A Rise in Incidence. Indian Journal of Otolaryngology and Head \& Neck Surgery. 2015;68(2):141-148. 